\title{
Comportamiento del alumnado universitario ante el desarrollo de su carrera profesional
}

\author{
José Tomás Bethencourt Benítez ${ }^{1}$, Lidia Cabrera Pérez ${ }^{2}$ \\ ${ }^{1}$ Departamento de Psicología Evolutiva y de la Educación, \\ Universidad de La Laguna, Tenerife \\ ${ }^{2}$ Departamento de Didáctica e Investigación Educativa, \\ Universidad de La Laguna, Tenerife
}

\section{Canarias, España}

jbethen@ull.es

Correspondencia: Dr. José Tomás Bethencourt Benítez. Universidad de La Laguna, Dpto de Psicología Evolutiva y de la Educación. Facultad de Psicología, Campus de Guajara. Tenerife. 38071. Canarias. E-mail: jbet$\underline{\text { hen@ull.es }}$

(C) Education \& Psychology $\mathrm{I}+\mathrm{D}+\mathrm{i}$ and Editorial EOS (Spain) 


\section{Resumen}

Introducción. Partiendo del modelo de desarrollo vocacional de Super y de las consideraciones de Salomone sobre la no variación teórica del modelo superiano durante 40 años, el presente trabajo aborda el estudio y caracterización del comportamiento vocacional del estudiantado universitario que se encuentra al término de su formación inicial. Más concretamente, el propósito de esta investigación fue doble. Por un lado, explorar el comportamiento general del alumnado ante su inmediato futuro profesional y, por otro lado, demostrar la existencia de diferencias en dicho comportamiento en función de algunas variables biográficas como la edad, el género, el curso o el tipo de titulación cursada.

Método. Para ello fueron seleccionados aleatoriamente 446 estudiantes de la Universidad de La Laguna pertenecientes al último o penúltimo curso de un total de 19 carreras, quienes tuvieron que contestar con las opciones de respuesta: (A) es exactamente igual o se parece mucho; (B) es lo contrario, no se parece nada; y (C) no lo considero, no he pensado en ello, a diversos ítems de un cuestionario referidos a: (1) Criterios de elección de las asignaturas optativas; (2) Prioridad de los planes profesionales; (3) Conocimiento del mercado laboral y de la profesión; y (4) Estrategias de búsqueda de empleo.

Resultados. Los resultados demuestran, por un lado, la existencia de ciertos niveles de inmadurez en la conducta vocacional del estudiantado que llega al término de la etapa formativa universitaria y, por otro lado, los análisis comparativos entre el estudiantado atendiendo a las variables biográficas arrojan diferencias según la edad, el género y la titulación, pero no en función del curso.

Conclusiones. Finalmente, de cara a futuras investigaciones, se sugiere que para ahondar en el conocimiento del desarrollo vocacional de los jóvenes universitarios, se necesita contar con mayor cantidad de estudios que permitan examinar las características generales y diferenciales de la conducta vocacional en las etapas de "establecimiento" y "mantenimiento" propuestas por Super, en las cuales los universitarios se encuentran ya plenamente integrados en el mercado laboral.

Palabras Clave: Conducta vocacional, desarrollo vocacional, enseñanza universitaria, transición formación-empleo, variables biográficas. 


\begin{abstract}
Introduction. Departing from the model of vocational development of Super and from Salomone's considerations on not theoretical variation of the Super model for 40 years, the present work approaches the study and characterization of the vocational behavior of the university students that one finds at the conclusion of your initial training. More concretely, the intention of this research was double, on one hand, to explore the general behavior of the students before your immediate professional future and, on the other hand, to demonstrate the difference existence in the above mentioned behavior depending on some biographical variables as the age, the gender, the course or the degree.
\end{abstract}

Method. For it there were selected at random 446 students of the La Laguna University belonging to the last or one before last course of a whole of 19 careers, who had to answer with the options of response: (A) is exactly equal or looks alike greatly; (B) is the opposite, does not look alike at all; and (C) I do not consider it, have not thought about it; to diverse items of a questionnaire recounted to: (1) Criteria of choice of the optional subjects; (2) Priority of the professional plans; (3) Knowledge of the labour market and of the profession; and (4) Strategies of search of employment.

Results. The results demonstrate, on one hand, the existence of certain levels of immaturity in the vocational behavior of the students that comes at the conclusion of the formative university stage and, on the other hand, the comparative analyses among the students attending to the biographical variables they throw differences according to the age, the gender and the degree, but not depending on the course.

Conclussions. Finally, with a view to future research, it is suggested that to go deeply into the knowledge of the vocational development of the all students, it is necessary rely on major quantity of studies that they should allow to examine the general and differential characteristics of the vocational behavior in the stages of "establishment" and "maintenance" proposed for Super, to which the university students are already fully integrated on the labour market.

Keywords: Vocational behavior, career development, higher education, training-employment transition, biographical variables.

Received: 08/02/08 Initial Acceptance: 11/02/08_ Definitive Acceptance: 15/05/08 


\section{Introducción}

En el desarrollo vocacional de los estudiantes universitarios, la terminación de su formación inicial y el paso a la profesionalización o laboralización activa constituye una de las transiciones vitales de mayor trascendencia. Desde que Super (1953) formulara su modelo en cinco etapas del desarrollo de la conducta vocacional, crecimiento, exploración, establecimiento, mantenimiento y declive, han transcurrido muchos años y la investigación sobre el desarrollo vocacional no ha cesado. Tal como ha señalado Salomone (1996) durante 40 años las proposiciones teóricas de Super no han cambiado sustancialmente.

El propio Super (1980) revisa y reformula su teoría originaria del año 1953 introduciendo planteamientos más dinámicos y evolutivos, que enfatizan una perspectiva centrada en el ciclo vital (life-span) y en el espacio vital (life-space) del desarrollo vocacional. Para Super (1953), la etapa de exploración estaría subdividida en las tres fases de fantasía, tentativa y realista. En tal sentido, el estudiantado universitario de último año de carrera se encontraría ubicado principalmente en la etapa segunda, exploración (15 a 24 años), dada su edad y grado de desarrollo de su conducta vocacional. En pocas palabras, las características psicológicas de la conducta vocacional en esa etapa evolutiva de la exploración son el autoanálisis, la búsqueda de información vocacional, y el análisis de los roles y actividades profesionales adecuadas a sí mismo.

La investigación que se ha publicado desde que Super enunciara su teoría del desarrollo vocacional es abundante. Desde entonces, las teorías del desarrollo vocacional basadas en etapas se han visto complementadas por aquellas centradas en las transiciones vocacionales y vitales (Rocabert, 2003). En éstas se sostiene que el desarrollo está también fuertemente asociado al contexto en el que se produce. Desde esta perspectiva de la transición, no cabe duda de que el momento en el que se encuentra el alumnado universitario de último curso de carrera impone ciertas condiciones, como son las de prolongar la formación inicial, comenzar la andadura laboral, o combinar ambas.

El impacto de la teoría de Super a nivel mundial ha llevado a Valls y Martínez (2004) a revisar las adaptaciones en castellano de su instrumento evaluativo el CDI (Career Development Inventory), a realizar una nueva adaptación para controlar su fiabilidad y validez, y a 
sugerir la conveniencia de trabajar en la elaboración de un nuevo instrumento y medida de la madurez vocacional adaptada al contexto educativo y ocupacional del Estado Español.

El interés científico y la utilidad práctica de los estudios sobre desarrollo vocacional queda patente, entre otras razones, por la gran proliferación de publicaciones referidas a programas para promover el desarrollo de la carrera de personas de diferentes edades (Álvarez, 1994; Rodríguez, 1995; Salvador y Peiró, 1986).

La transición de los universitarios desde la formación al empleo ha sido objeto de considerable atención, pudiéndose destacar la encuesta CHEERS (Career after Higher Education: a European Research Study) de cuyos datos han surgido publicaciones como la de GarcíaMontalvo (2001), en la que se analiza la transición hacia el mercado laboral de forma diferencial, esto es, en función de variables como nacionalidad, rama o campo de estudio, género, o tipo de titulación (ciclo corto o largo), pero no atendiendo a otras variables como edad.

En los estudios sobre el desarrollo vocacional no se ha prestado la suficiente atención a las diferencias evolutivas de la conducta vocacional, en función de diversidad de variables tanto personales como contextuales. En tal sentido y a modo de ejemplo, podemos citar algunas investigaciones previas. Rísquez, (2004) encuentra que las diferencias culturales entre irlandeses y españoles hacen que los primeros tengan una motivación hacia el trabajo más positiva. Rocabert, Rivas y Pascual (2000) obtienen diferencias en el desarrollo vocacional de estudiantes universitarios pertenecientes al grupo vocacional deportivo en función de los distintos itinerarios curriculares que se sigan.

El propósito pues de la presente investigación es el de explorar y caracterizar el comportamiento vocacional del estudiantado universitario que se encuentra al final de su formación inicial, y demostrar la existencia de diferencias en el mismo, en función de variables biográficas como la edad, el género, el curso o el tipo de titulación cursada.

\section{Método}

\section{Participantes}

La muestra de la presente investigación estuvo constituida por 446 estudiantes de la Universidad de La Laguna seleccionados aleatoriamente. Algunas de las características bio- 
gráficas del estudiantado analizado fueron las siguientes: en cuanto al tipo de titulación cursada el $56.5 \%$ estaba en Licenciaturas y el $43.5 \%$ en Diplomaturas; el $69.5 \%$ son mujeres; las edades oscilaban desde 19 hasta 47 años con una media de 23 años; el 80.5\% estaba en el último curso de su carrera y el 19.5\% en el penúltimo curso; las titulaciones a las que pertenecían los estudiantes de la muestra fueron por orden alfabético las siguientes: Administración y Dirección de Empresas, Arquitectura Técnica, Bellas Artes, Biología, Derecho, Enfermería, Fisioterapia, Geografía, Ingeniería Informática, Ingeniería Técnica en Informática de Gestión, Logopedia, Maestro, Matemáticas, Psicología, Psicopedagogía, Química, Sociología, Trabajo Social y Turismo.

\section{Instrumento}

Para la recogida de los datos empleamos un cuestionario que incluía dos tipos de ítems. Unos referidos a las características biográficas anteriormente señaladas, y otros, que recogían información relativa a: (1) Criterios de elección de las asignaturas optativas; (2) Prioridad de los planes profesionales; (3) Conocimiento del mercado laboral y de la profesión; y (4) Estrategias de búsqueda de empleo.

Los ítems propios de la conducta vocacional iban precedidos de un encabezado que literalmente decía: "Estos enunciados describen distintos aspectos o formas de actuar que tienen que ver con decisiones que has ido tomando a lo largo de la carrera, o que tendrás que realizar próximamente. Responde redondeando la opción $A, B$ o C que mejor refleje tu caso: (A) es exactamente igual o se parece mucho; (B) es lo contrario, no se parece nada; y (C) no lo considero, no he pensado en ello".

\section{Procedimiento}

El cuestionario fue aplicado colectivamente en los grupos naturales de clase por cinco alumnas de Psicopedagogía debidamente entrenadas como encuestadoras ${ }^{1}$. La fecha de recogida de los datos fue durante los meses de marzo a mayo del curso académico. El estudiantado encuestado debía responder solamente a una de las tres opciones de respuesta para cada ítem. Los datos obtenidos fueron sometidos a procesamiento estadístico con el programa SPSS versión 14.0 para Windows.

\footnotetext{
${ }^{1}$ Estas alumnas fueron Artemisa Barreto Espino, Piedad García Piñero, Mercedes Hernández Pedreira, Mónica Montesdeoca Bosa, Omaira Pérez García.
} 


\section{Resultados}

Los resultados obtenidos son presentados primeramente para la muestra total y, posteriormente, atendiendo a las diferencias intra en función de algunas variables biográficas como edad, género, titulación y curso. En la tabla 1 mostramos las respuestas dadas por la muestra total a los distintos ítems del cuestionario.

Tabla 1. Porcentajes de respuestas en la muestra total

\begin{tabular}{|c|c|c|c|}
\hline Ítems & A & B & $\mathbf{C}$ \\
\hline Elijo las asignaturas que tienen fama de fáciles & 34.4 & 32.6 & 33.0 \\
\hline Al matricularme, busco sobre todo el horario más cómodo & 51.5 & 26.7 & 21.8 \\
\hline Aunque me interese la materia, procuro evitar a los profesores que son muy duros & 24.4 & 44.2 & 31.4 \\
\hline No me importa qué asignatura cursar, si la puedo hacer junto con mis amigos & 10.1 & 50.1 & 39.8 \\
\hline Elijo aquellas asignaturas que más me interesan, gustan o mejor me van & 83.3 & 10.0 & 6.7 \\
\hline $\begin{array}{l}\text { Al elegir materias procuro averiguar las consecuencias para mi futura carrera profesional o } \\
\text { especialidad }\end{array}$ & 64.9 & 13.9 & 21.2 \\
\hline Compararme con mis compañeros, me sirve para aceptar mis limitaciones y posibilidades & 29.7 & 34.2 & 36.1 \\
\hline $\begin{array}{l}\text { Estoy dispuesto durante unos años, si es preciso, a demorar mis planes personales con tal de } \\
\text { conseguir profesionalmente lo que quiero }\end{array}$ & 52.4 & 24.7 & 22.9 \\
\hline En este momento de mi vida, dominan sobre todo lo demás, mis planes de carrera profesional & 54.5 & 29.1 & 16.4 \\
\hline Estoy adquiriendo formación complementaria a la carrera (prácticas, informática, idiomas...) & 49.7 & 29.2 & 21.1 \\
\hline Conozco bastante bien el mundo laboral o académico al que me estoy dirigiendo & 44.4 & 36.2 & 19.4 \\
\hline Estoy al tanto de las convocatorias de becas, estancias en el extranjero, contactos, etc. & 35.1 & 36.0 & 28.9 \\
\hline $\begin{array}{l}\text { La mayoría de libros, manuales, etc. que manejo, están relacionados con la profesión que } \\
\text { quiero ejercer }\end{array}$ & 63.8 & 20.6 & 15.6 \\
\hline Me encuentro capacitado para realizar una entrevista de trabajo & 53.1 & 23.8 & 23.1 \\
\hline $\begin{array}{l}\text { Conozco las diferentes técnicas y recursos para buscar empleo (preparar currículum, carta de } \\
\text { presentación...) }\end{array}$ & 52.9 & 30.2 & 16.9 \\
\hline Tengo planificado lo que voy a hacer para conseguir el puesto de trabajo que quiero & 44.4 & 28.5 & 27.1 \\
\hline $\begin{array}{l}\text { Sé a quien puedo acudir y cómo puedo establecer los contactos necesarios para conseguir el } \\
\text { puesto de trabajo que quiero }\end{array}$ & 29.6 & 40.2 & 30.3 \\
\hline $\begin{array}{l}\text { Tengo muy en cuenta las tendencias del mercado laboral y profesional para decidir la espe- } \\
\text { cialización o puesto de trabajo que quiero }\end{array}$ & 40.1 & 29.8 & 30.1 \\
\hline Sé analizar las ofertas de empleo y relacionarlas con mis posibilidades & 52.2 & 22.0 & 25.8 \\
\hline $\begin{array}{l}\text { Sé dónde buscar información sobre puestos de trabajo que se pueden ajustar a mi preparación } \\
\text { y preferencias }\end{array}$ & 45.4 & 32.7 & 21.9 \\
\hline $\begin{array}{l}\text { Conozco el tipo de tareas que hay que realizar en la profesión o puesto de trabajo que espero } \\
\text { ejercer }\end{array}$ & 62.2 & 22.9 & 14.9 \\
\hline $\begin{array}{l}\text { Tengo un buen conocimiento de las condiciones laborales (sueldo, contratos, promoción, } \\
\text { seguridad, etc.) que puedo obtener al inicio de mi profesión }\end{array}$ & 33.33 & 37.9 & 28.8 \\
\hline Conozco los recursos necesarios para elaborar un plan de autoempleo & 11.2 & 44.7 & 44.1 \\
\hline
\end{tabular}
(A) es exactamente igual o se parece mucho
(B) es lo contrario, no se parece nada
(C) no lo considero, no he pensado en ello

\section{Criterios de elección de asignaturas optativas}

La elección de asignaturas optativas en el ámbito de la enseñanza universitaria es realizada primeramente atendiendo a los intereses vocacionales (83.3\%), en segundo lugar teniendo en cuenta las consecuencias futuras para el desarrollo de la carrera profesional 
(64.9\%), en tercer lugar según el horario (51.5\%), y en cuarto lugar, considerando la dificultad (34.4\%). Por otra parte, debe resaltarse la baja incidencia que el efecto de arrastre de los amigos tiene en el estudiantado universitario a la hora de elegir optativas (10.1\%).

\section{Prioridad de los planes profesionales}

Más de la mitad de los estudiantes encuestados afirman que sus planes profesionales son prioritarios (54.5\%) y que están dispuestos a demorar otros planes personales para darles preferencia a los planes profesionales $(52.4 \%)$.

\section{Conocimiento del mercado laboral y de la profesión}

El 63.8\% de los encuestados afirma que la mayoría de las publicaciones y otro tipo de información impresa que maneja está relacionada con la profesión que desea ejercer. El $62.2 \%$ de los estudiantes declara poseer un buen conocimiento de las tareas que hay que realizar en la profesión o puesto de trabajo que espera ejercer. Casi la mitad del estudiantado (49.7\%) reconoce encontrarse en un proceso de adquisición de formación complementaria a la carrera. Menos de la mitad de los universitarios (44.4\%) conoce bien el mercado laboral o el mundo académico. El interés y preocupación por las becas, estancias en el extranjero o contactos es baja (35.1\%). Finalmente, tan solo un tercio de los encuestados (33.3\%) reconoce poseer un buen conocimiento de las condiciones laborales imperantes en el ejercicio de la profesión deseada, relativas a sueldo, contratos, promoción, o seguridad.

\section{Estrategias de búsqueda de empleo}

Más de la mitad del estudiantado (53.1\%) se considera capacitado para hacer frente a una entrevista de trabajo. El 52.9\% conoce las diferentes estrategias para buscar empleo. El $52.2 \%$ sabe analizar las ofertas de empleo y relacionarlas con sus posibilidades. Menos de la mitad del estudiantado (45.4\%) sabe dónde buscar información sobre puestos de trabajo que se puedan ajustar a su preparación y preferencias. El 44.4\% tiene planificado lo que va a hacer para conseguir el puesto de trabajo deseado. El 40.1\% tiene muy en cuenta las tendencias del mercado laboral y profesional para decidir la especialización o puesto de trabajo que quiere. Resulta llamativo que tan solo el $29.6 \%$ de los estudiantes sepa a quién poder acudir y cómo poder establecer los contactos necesarios para conseguir el puesto de trabajo al que aspiran. Por último, una minoría (11.2\%) de estudiantes conoce los recursos necesarios para elaborar un plan de autoempleo. 


\section{Diferencias en el comportamiento vocacional según la edad}

La muestra fue dividida en dos grupos, el primero formado por el 55.5\% con 23 o menos años de edad y el segundo por el $44.5 \%$ con 24 o más años. Los datos fueron sometidos con el SPSS a un análisis de diferencias de medias para muestras independientes. En la tabla 2 puede observarse que en general las diferencias entre los estudiantes de baja edad y alta edad son pocas y cuando se producen lo hacen tan solo al más bajo nivel de confianza ( $p<0.05)$, pero no al nivel medio $(\mathrm{p}<0.01)$ o alto $(\mathrm{p}<0.001)$.

Las diferencias encontradas estriban en que el estudiantado de más edad evita al profesorado exigente, se deja arrastrar menos por los amigos, elige asignaturas concediéndole menor importancia a sus intereses vocacionales, está menos dispuesto a demorar sus planes personales en favor de los planes profesionales, se siente más capacitado para encarar una entrevista de trabajo, posee un mayor conocimiento de las estrategias de búsqueda de empleo y de los contactos necesarios para conseguir empleo, sabe mejor cómo buscar información sobre los puestos de trabajo interesantes, y finalmente, tiene un mayor conocimiento del autoempleo como alternativa laboral.

\section{Diferencias en el comportamiento vocacional según el género}

Las diferencias entre hombres y mujeres son más escasas que las habidas para la variable edad, pero de mayor magnitud. Nueve son las diferencias encontradas según la edad y cuatro en el caso de la variable género (tabla 3 ).

Los resultados aquí señalan que los hombres tienen menos en cuenta sus intereses vocacionales al elegir asignaturas, prestan menos atención a las consecuencias profesionales de las asignaturas elegidas, en cambio, se fijan más en las tendencias del mercado laboral a la hora de decidir especialidad u ocupación y, por último, tienen un mayor conocimiento de las condiciones laborales (sueldo, contratos, promoción, etc.) de las ocupaciones que les interesan. 
Tabla 2. Diferencias de media según edad

\begin{tabular}{|c|c|c|c|}
\hline Ítems & $\begin{array}{l}\text { Media } \\
\text { baja } \\
\text { Edad }\end{array}$ & $\begin{array}{l}\text { Media } \\
\text { alta } \\
\text { Edad }\end{array}$ & Sig. \\
\hline Elijo las asignaturas que tienen fama de fáciles & 0.52 & 0.52 & 0.970 \\
\hline Al matricularme, busco sobre todo el horario más cómodo & 0.62 & 0.71 & 0.110 \\
\hline $\begin{array}{l}\text { Aunque me interese la materia, procuro evitar a los profesores que son muy } \\
\text { duros }\end{array}$ & 0.30 & 0.42 & $0.038^{*}$ \\
\hline $\begin{array}{l}\text { No me importa qué asignatura cursar, si la puedo hacer junto con mis ami- } \\
\text { gos }\end{array}$ & 0.19 & 0.15 & $0.031 *$ \\
\hline Elijo aquellas asignaturas que más me interesan, gustan o mejor me van & 0.91 & 0.87 & $0.032 *$ \\
\hline $\begin{array}{l}\text { Al elegir materias procuro averiguar las consecuencias para mi futura ca- } \\
\text { rrera profesional o especialidad }\end{array}$ & 0.86 & 0.78 & 0.084 \\
\hline $\begin{array}{l}\text { Compararme con mis compañeros, me sirve para aceptar mis limitaciones } \\
\text { y posibilidades }\end{array}$ & 0.45 & 0.45 & 0.998 \\
\hline $\begin{array}{l}\text { Estoy dispuesto durante unos años, si es preciso, a demorar mis planes } \\
\text { personales con tal de conseguir profesionalmente lo que quiero }\end{array}$ & 0.72 & 0.60 & $0.017 *$ \\
\hline $\begin{array}{l}\text { En este momento de mi vida, dominan sobre todo lo demás, mis planes de } \\
\text { carrera profesional }\end{array}$ & 0.64 & 0.65 & 0.821 \\
\hline $\begin{array}{l}\text { Estoy adquiriendo formación complementaria a la carrera (prácticas, in- } \\
\text { formática, idiomas...) }\end{array}$ & 0.62 & 0.63 & 0.878 \\
\hline $\begin{array}{l}\text { Conozco bastante bien el mundo laboral o académico al que me estoy diri- } \\
\text { giendo }\end{array}$ & 0.52 & 0.59 & 0.199 \\
\hline $\begin{array}{l}\text { Estoy al tanto de las convocatorias de becas, estancias en el extranjero, } \\
\text { contactos, etc. }\end{array}$ & 0.49 & 0.49 & 0.918 \\
\hline $\begin{array}{l}\text { La mayoría de libros, manuales, etc. que manejo, están relacionados con la } \\
\text { profesión que quiero ejercer }\end{array}$ & 0.73 & 0.78 & 0.267 \\
\hline Me encuentro capacitado para realizar una entrevista de trabajo & 0.64 & 0.75 & $0.029 *$ \\
\hline $\begin{array}{l}\text { Conozco las diferentes técnicas y recursos para buscar empleo (preparar } \\
\text { currículum, carta de presentación...) }\end{array}$ & 0.58 & 0.71 & $0.016^{*}$ \\
\hline $\begin{array}{l}\text { Tengo planificado lo que voy a hacer para conseguir el puesto de trabajo } \\
\text { que quiero }\end{array}$ & 0.57 & 0.63 & 0.301 \\
\hline $\begin{array}{l}\text { Sé a quien puedo acudir y cómo puedo establecer los contactos necesarios } \\
\text { para conseguir el puesto de trabajo que quiero }\end{array}$ & 0.36 & 0.48 & $0.040 *$ \\
\hline $\begin{array}{l}\text { Tengo muy en cuenta las tendencias del mercado laboral y profesional para } \\
\text { decidir la especialización o puesto de trabajo que quiero }\end{array}$ & 0.56 & 0.58 & 0.735 \\
\hline Sé analizar las ofertas de empleo y relacionarlas con mis posibilidades & 0.66 & 0.76 & 0.075 \\
\hline $\begin{array}{l}\text { Sé dónde buscar información sobre puestos de trabajo que se pueden ajus- } \\
\text { tar a mi preparación y preferencias }\end{array}$ & 0.53 & 0.64 & $0.041 *$ \\
\hline $\begin{array}{l}\text { Conozco el tipo de tareas que hay que realizar en la profesión o puesto de } \\
\text { trabajo que espero ejercer }\end{array}$ & 0.72 & 0.75 & 0.560 \\
\hline $\begin{array}{l}\text { Tengo un buen conocimiento de las condiciones laborales (sueldo, contra- } \\
\text { tos, promoción, seguridad, etc.) que puedo obtener al inicio de mi profe- } \\
\text { sión }\end{array}$ & 0.44 & 0.50 & 0.344 \\
\hline Conozco los recursos necesarios para elaborar un plan de autoempleo & 0.14 & 0.27 & $0.017 *$ \\
\hline
\end{tabular}


Tabla 3. Diferencias de media según género

\begin{tabular}{|c|c|c|c|}
\hline Ítems & $\begin{array}{l}\text { Media } \\
\text { Hombre }\end{array}$ & $\begin{array}{l}\text { Media } \\
\text { Mujer }\end{array}$ & Sig. \\
\hline Elijo las asignaturas que tienen fama de fáciles & 0.48 & 0.53 & 0.404 \\
\hline Al matricularme, busco sobre todo el horario más cómodo & 0.60 & 0.68 & 0.196 \\
\hline $\begin{array}{l}\text { Aunque me interese la materia, procuro evitar a los profesores que son muy } \\
\text { duros }\end{array}$ & 0.42 & 0.33 & 0.146 \\
\hline $\begin{array}{l}\text { No me importa qué asignatura cursar, si la puedo hacer junto con mis ami- } \\
\text { gos }\end{array}$ & 0.20 & 0.16 & 0.460 \\
\hline Elijo aquellas asignaturas que más me interesan, gustan o mejor me van & 0.82 & 0.92 & $0.020 *$ \\
\hline $\begin{array}{l}\text { Al elegir materias procuro averiguar las consecuencias para mi futura ca- } \\
\text { rrera profesional o especialidad }\end{array}$ & 0.71 & 0.88 & $\begin{array}{l}0.001 \\
* * *\end{array}$ \\
\hline $\begin{array}{l}\text { Compararme con mis compañeros, me sirve para aceptar mis limitaciones } \\
\text { y posibilidades }\end{array}$ & 0.46 & 0.46 & 0.980 \\
\hline $\begin{array}{l}\text { Estoy dispuesto durante unos años, si es preciso, a demorar mis planes } \\
\text { personales con tal de conseguir profesionalmente lo que quiero }\end{array}$ & 0.63 & 0.70 & 0.225 \\
\hline $\begin{array}{l}\text { En este momento de mi vida, dominan sobre todo lo demás, mis planes de } \\
\text { carrera profesional }\end{array}$ & 0.69 & 0.63 & 0.307 \\
\hline $\begin{array}{l}\text { Estoy adquiriendo formación complementaria a la carrera (prácticas, in- } \\
\text { formática, idiomas...) }\end{array}$ & 0.63 & 0.62 & 0.911 \\
\hline $\begin{array}{l}\text { Conozco bastante bien el mundo laboral o académico al que me estoy diri- } \\
\text { giendo }\end{array}$ & 0.58 & 0.55 & 0.680 \\
\hline $\begin{array}{l}\text { Estoy al tanto de las convocatorias de becas, estancias en el extranjero, } \\
\text { contactos, etc. }\end{array}$ & 0.43 & 0.52 & 0.162 \\
\hline $\begin{array}{l}\text { La mayoría de libros, manuales, etc. que manejo, están relacionados con la } \\
\text { profesión que quiero ejercer }\end{array}$ & 0.78 & 0.74 & 0.412 \\
\hline Me encuentro capacitado para realizar una entrevista de trabajo & 0.74 & 0.67 & 0.226 \\
\hline $\begin{array}{l}\text { Conozco las diferentes técnicas y recursos para buscar empleo (preparar } \\
\text { currículum, carta de presentación...) }\end{array}$ & 0.63 & 0.65 & 0.798 \\
\hline $\begin{array}{l}\text { Tengo planificado lo que voy a hacer para conseguir el puesto de trabajo } \\
\text { que quiero }\end{array}$ & 0.56 & 0.62 & 0.391 \\
\hline $\begin{array}{l}\text { Sé a quien puedo acudir y cómo puedo establecer los contactos necesarios } \\
\text { para conseguir el puesto de trabajo que quiero }\end{array}$ & 0.47 & 0.40 & 0.275 \\
\hline $\begin{array}{l}\text { Tengo muy en cuenta las tendencias del mercado laboral y profesional para } \\
\text { decidir la especialización o puesto de trabajo que quiero }\end{array}$ & 0.68 & 0.52 & $\begin{array}{l}0.009 \\
* *\end{array}$ \\
\hline Sé analizar las ofertas de empleo y relacionarlas con mis posibilidades & 0.75 & 0.68 & 0.224 \\
\hline $\begin{array}{l}\text { Sé dónde buscar información sobre puestos de trabajo que se pueden ajus- } \\
\text { tar a mi preparación y preferencias }\end{array}$ & 0.64 & 0.56 & 0.155 \\
\hline $\begin{array}{l}\text { Conozco el tipo de tareas que hay que realizar en la profesión o puesto de } \\
\text { trabajo que espero ejercer }\end{array}$ & 0.75 & 0.73 & 0.636 \\
\hline $\begin{array}{l}\text { Tengo un buen conocimiento de las condiciones laborales (sueldo, contra- } \\
\text { tos, promoción, seguridad, etc.) que puedo obtener al inicio de mi profe- } \\
\text { sión }\end{array}$ & 0.62 & 0.41 & $\begin{array}{l}0.001 \\
* * *\end{array}$ \\
\hline Conozco los recursos necesarios para elaborar un plan de autoempleo & 0.23 & 0.19 & 0.495 \\
\hline
\end{tabular}




\section{Diferencias en el comportamiento vocacional según la titulación}

En la tabla 4 presentamos las medias de los grupos de estudiantes que cursaban titulaciones de nivel licenciatura y nivel diplomatura, encontrándose que tan solo en cuatro de los veintitrés ítems se producen diferencias estadísticamente significativas.

El estudiantado que cursa licenciaturas afirma que compararse con sus compañeros le sirve más para aceptar sus limitaciones y posibilidades, conoce peor el mundo laboral o académico, se fija menos en las tendencias del mercado laboral a la hora de decidir especialidad u ocupación y, finalmente, tiene menos conocimiento del tipo de tareas que hay que realizar en la profesión o puesto de trabajo que espera ejercer.

\section{Diferencias en el comportamiento vocacional según el curso}

En la tabla 5 se exponen las medias para el grupo de estudiantes que se encontraban cursando el último curso de su carrera universitaria frente a los del penúltimo curso. Los datos no arrojan diferencias en función de esta variable sobre el comportamiento vocacional, lo que nos indica que el tramo temporal de un curso es insuficiente para que se produzcan cambios en la madurez vocacional.

\section{Discusión}

En el comportamiento general del estudiantado universitario ante el desarrollo de su carrera profesional se observan claros contrastes y evidentes desigualdades, que ponen de relieve la existencia de ciertos niveles de inmadurez con los que se llega al término de la etapa formativa universitaria. Esta conclusión se deriva, entre otros, de los datos relativos al porcentaje de estudiantes que eligen la opción de respuesta (C) "no lo considero, no he pensado en ello", para algunos de los aspectos importantes concernientes con su futuro profesional.

En la toma de decisiones académicas del estudiantado universitario se combinan criterios superficiales y comprometidos con la carrera, tal como ya hemos constatado en otras investigaciones, tanto para el caso de elección de asignaturas optativas, como para el de créditos de libre elección (Álvarez, Feliciano, Cabrera, Bethencourt y González, 2000; González, Álvarez, Cabrera y Bethencourt, 2002). En estas decisiones el estudiantado trata de conciliar sus intereses y preferencias con la dificultad de las materias y los horarios docentes. La preeminencia de los intereses vocacionales también ha quedado demostrada en otros estudios 
sobre el proceso de elección de carrera universitaria. Concretamente en el estudio de Bethencourt y col. (2005) mostró ser la variable más influyente.

Tabla 4. Diferencias de media según titulación

\begin{tabular}{|c|c|c|c|}
\hline Ítems & \begin{tabular}{|l|} 
Media \\
Licenciatura
\end{tabular} & \begin{tabular}{|l|} 
Media \\
Diplomatura \\
\end{tabular} & Sig. \\
\hline Elijo las asignaturas que tienen fama de fáciles & 0.52 & 0.51 & 0.947 \\
\hline Al matricularme, busco sobre todo el horario más cómodo & 0.64 & 0.67 & 0.557 \\
\hline $\begin{array}{l}\text { Aunque me interese la materia, procuro evitar a los profesores que son } \\
\text { muy duros }\end{array}$ & 0.38 & 0.31 & 0.272 \\
\hline $\begin{array}{l}\text { No me importa qué asignatura cursar, si la puedo hacer junto con mis } \\
\text { amigos }\end{array}$ & 0.16 & 0.19 & 0.490 \\
\hline Elijo aquellas asignaturas que más me interesan, gustan o mejor me van & 0.89 & 0.91 & 0.431 \\
\hline $\begin{array}{l}\text { Al elegir materias procuro averiguar las consecuencias para mi futura } \\
\text { carrera profesional o especialidad }\end{array}$ & 0.82 & 0.84 & 0.627 \\
\hline $\begin{array}{l}\text { Compararme con mis compañeros, me sirve para aceptar mis limitacio- } \\
\text { nes y posibilidades }\end{array}$ & 0.53 & 0.39 & $0.027 *$ \\
\hline $\begin{array}{l}\text { Estoy dispuesto durante unos años, si es preciso, a demorar mis planes } \\
\text { personales con tal de conseguir profesionalmente lo que quiero }\end{array}$ & 0.68 & 0.67 & 0.975 \\
\hline $\begin{array}{l}\text { En este momento de mi vida, dominan sobre todo lo demás, mis planes } \\
\text { de carrera profesional }\end{array}$ & 0.68 & 0.62 & 0.269 \\
\hline $\begin{array}{l}\text { Estoy adquiriendo formación complementaria a la carrera (prácticas, } \\
\text { informática, idiomas...) }\end{array}$ & 0.62 & 0.64 & 0.686 \\
\hline $\begin{array}{l}\text { Conozco bastante bien el mundo laboral o académico al que me estoy } \\
\text { dirigiendo }\end{array}$ & 0.49 & 0.64 & $\begin{array}{l}0.004 \\
* *\end{array}$ \\
\hline $\begin{array}{l}\text { Estoy al tanto de las convocatorias de becas, estancias en el extranjero, } \\
\text { contactos, etc. }\end{array}$ & 0.48 & 0.51 & 0.591 \\
\hline $\begin{array}{l}\text { La mayoría de libros, manuales, etc. que manejo, están relacionados con } \\
\text { la profesión que quiero ejercer }\end{array}$ & 0.72 & 0.79 & 0.152 \\
\hline Me encuentro capacitado para realizar una entrevista de trabajo & 0.66 & 0.73 & 0.187 \\
\hline $\begin{array}{l}\text { Conozco las diferentes técnicas y recursos para buscar empleo (preparar } \\
\text { currículum, carta de presentación...) }\end{array}$ & 0.62 & 0.64 & 0.694 \\
\hline $\begin{array}{l}\text { Tengo planificado lo que voy a hacer para conseguir el puesto de trabajo } \\
\text { que quiero }\end{array}$ & 0.63 & 0.59 & 0.488 \\
\hline $\begin{array}{l}\text { Sé a quien puedo acudir y cómo puedo establecer los contactos necesa- } \\
\text { rios para conseguir el puesto de trabajo que quiero }\end{array}$ & 0.39 & 0.48 & 0.127 \\
\hline $\begin{array}{l}\text { Tengo muy en cuenta las tendencias del mercado laboral y profesional } \\
\text { para decidir la especialización o puesto de trabajo que quiero }\end{array}$ & 0.51 & 0.65 & $0.013 *$ \\
\hline Sé analizar las ofertas de empleo y relacionarlas con mis posibilidades & 0.73 & 0.66 & 0.178 \\
\hline $\begin{array}{l}\text { Sé dónde buscar información sobre puestos de trabajo que se pueden } \\
\text { ajustar a mi preparación y preferencias }\end{array}$ & 0.56 & 0.60 & 0.534 \\
\hline $\begin{array}{l}\text { Conozco el tipo de tareas que hay que realizar en la profesión o puesto } \\
\text { de trabajo que espero ejercer }\end{array}$ & 0.66 & 0.81 & $\begin{array}{l}0.001 \\
* * *\end{array}$ \\
\hline $\begin{array}{l}\text { Tengo un buen conocimiento de las condiciones laborales (sueldo, con- } \\
\text { tratos, promoción, seguridad, etc.) que puedo obtener al inicio de mi } \\
\text { profesión }\end{array}$ & 0.45 & 0.50 & 0.385 \\
\hline Conozco los recursos necesarios para elaborar un plan de autoempleo & 0.17 & 0.23 & 0.299 \\
\hline
\end{tabular}


Tabla 5. Diferencias de media según curso

\begin{tabular}{|c|c|c|c|}
\hline Ítems & $\begin{array}{l}\text { Media } \\
\text { Último }\end{array}$ & \begin{tabular}{|l|l|} 
Media \\
Penúltimo
\end{tabular} & Sig. \\
\hline Elijo las asignaturas que tienen fama de fáciles & 0.52 & 0.50 & 0.789 \\
\hline Al matricularme, busco sobre todo el horario más cómodo & 0.65 & 0.71 & 0.327 \\
\hline $\begin{array}{l}\text { Aunque me interese la materia, procuro evitar a los profesores que son } \\
\text { muy duros }\end{array}$ & 0.37 & 0.32 & 0.534 \\
\hline $\begin{array}{l}\text { No me importa qué asignatura cursar, si la puedo hacer junto con mis } \\
\text { amigos }\end{array}$ & 0.17 & 0.16 & 0.847 \\
\hline Elijo aquellas asignaturas que más me interesan, gustan o mejor me van & 0.89 & 0.89 & 0.879 \\
\hline $\begin{array}{l}\text { Al elegir materias procuro averiguar las consecuencias para mi futura } \\
\text { carrera profesional o especialidad }\end{array}$ & 0.82 & 0.84 & 0.660 \\
\hline $\begin{array}{l}\text { Compararme con mis compañeros, me sirve para aceptar mis limitaciones } \\
\text { y posibilidades }\end{array}$ & 0.47 & 0.43 & 0.615 \\
\hline $\begin{array}{l}\text { Estoy dispuesto durante unos años, si es preciso, a demorar mis planes } \\
\text { personales con tal de conseguir profesionalmente lo que quiero }\end{array}$ & 0.67 & 0.69 & 0.778 \\
\hline $\begin{array}{l}\text { En este momento de mi vida, dominan sobre todo lo demás, mis planes } \\
\text { de carrera profesional }\end{array}$ & 0.67 & 0.57 & 0.149 \\
\hline $\begin{array}{l}\text { Estoy adquiriendo formación complementaria a la carrera (prácticas, } \\
\text { informática, idiomas...) }\end{array}$ & 0.63 & 0.60 & 0.618 \\
\hline $\begin{array}{l}\text { Conozco bastante bien el mundo laboral o académico al que me estoy } \\
\text { dirigiendo }\end{array}$ & 0.56 & 0.52 & 0.608 \\
\hline $\begin{array}{l}\text { Estoy al tanto de las convocatorias de becas, estancias en el extranjero, } \\
\text { contactos, etc. }\end{array}$ & 0.48 & 0.54 & 0.395 \\
\hline $\begin{array}{l}\text { La mayoría de libros, manuales, etc. que manejo, están relacionados con } \\
\text { la profesión que quiero ejercer }\end{array}$ & 0.75 & 0.75 & 0.993 \\
\hline Me encuentro capacitado para realizar una entrevista de trabajo & 0.69 & 0.67 & 0.752 \\
\hline $\begin{array}{l}\text { Conozco las diferentes técnicas y recursos para buscar empleo (preparar } \\
\text { currículum, carta de presentación...) }\end{array}$ & 0.63 & 0.65 & 0.688 \\
\hline $\begin{array}{l}\text { Tengo planificado lo que voy a hacer para conseguir el puesto de trabajo } \\
\text { que quiero }\end{array}$ & 0.62 & 0.57 & 0.573 \\
\hline $\begin{array}{l}\text { Sé a quien puedo acudir y cómo puedo establecer los contactos necesa- } \\
\text { rios para conseguir el puesto de trabajo que quiero }\end{array}$ & 0.43 & 0.37 & 0.364 \\
\hline $\begin{array}{l}\text { Tengo muy en cuenta las tendencias del mercado laboral y profesional } \\
\text { para decidir la especialización o puesto de trabajo que quiero }\end{array}$ & 0.57 & 0.60 & 0.636 \\
\hline Sé analizar las ofertas de empleo y relacionarlas con mis posibilidades & 0.72 & 0.63 & 0.233 \\
\hline $\begin{array}{l}\text { Sé dónde buscar información sobre puestos de trabajo que se pueden } \\
\text { ajustar a mi preparación y preferencias }\end{array}$ & 0.58 & 0.58 & 0.958 \\
\hline $\begin{array}{l}\text { Conozco el tipo de tareas que hay que realizar en la profesión o puesto de } \\
\text { trabajo que espero ejercer }\end{array}$ & 0.73 & 0.71 & 0.693 \\
\hline $\begin{array}{l}\text { Tengo un buen conocimiento de las condiciones laborales (sueldo, con- } \\
\text { tratos, promoción, seguridad, etc.) que puedo obtener al inicio de mi } \\
\text { profesión }\end{array}$ & 0.46 & 0.47 & 0.928 \\
\hline Conozco los recursos necesarios para elaborar un plan de autoempleo & 0.21 & 0.17 & 0.552 \\
\hline
\end{tabular}


El bajo porcentaje de estudiantes $(29.6 \%)$ de la presente investigación que afirma saber a quién poder acudir y cómo poder establecer los contactos necesarios para conseguir el puesto de trabajo al que aspira, se nos revela como preocupante, si lo contemplamos desde la perspectiva de la enorme transcendencia que los titulados universitarios, ya ejercientes o incorporados al mercado laboral, conceden a las redes sociales y a los contactos personales, siendo éste el primer medio de consecución de empleo (Álvarez et al., 2000a; Bethencourt, Álvarez, Cabrera y González, 2000).

El bajísimo porcentaje (11.2\%) de estudiantes que conoce y se plantea el autoempleo como una alternativa de profesionalización, confirma la tendencia constante que se viene manteniendo en anteriores investigaciones, en las que se constata que el empleo por cuenta ajena es la modalidad mayoritaria de laboralización del colectivo de titulados universitarios, llegando al 95\% en el caso específico de los licenciados en Psicopedagogía (Álvarez et al., 2000b; Bethencourt, González, Álvarez y Cabrera, 2005).

El alto porcentaje $(52.4 \%)$ de estudiantes universitarios que en nuestra investigación manifiesta que está dispuesto a demorar sus planes personales para darle preferencia a los planes profesionales, nos lleva a considerar que nos encontramos ante un comportamiento positivo que previene y evita el abandono de opciones formativas, tal como han demostrado Cabrera, Bethencourt, González y Álvarez (2006). Estos autores constatan que la persistencia y perseverancia es la principal variable que influye en el no abandono de los estudios universitarios. En tal sentido, esa característica psicológica de los universitarios nos sitúa ante individuos capaces de demorar las recompensas, de superar obstáculos y dificultades, de mantener claras las metas de largo plazo, de fijar firmemente el rumbo o dirección de futuro y, en definitiva, de ser constantes en el mantenimiento de los planes establecidos.

Los análisis comparativos entre el estudiantado atendiendo a las variables biográficas arrojan diferencias según la edad, el género y la titulación, pero no en función del curso.

Las diferencias en el comportamiento vocacional del estudiantado universitario apuntan a una mayor madurez en los de edad superior, en los de diplomaturas, en las mujeres en algunos aspectos y en los hombres en otros aspectos. El estudiantado de las diplomaturas al estar cursando carreras de corta duración parece estar más próximo y comprometido con su futuro profesional, teniendo un conocimiento mayor del mercado laboral y de la profesión. 
Los hombres manifiestan un mayor pragmatismo y utilitarismo preocupándose por conocer mejor el mercado laboral, mientras que las mujeres toman decisiones más genuinamente vocacionales y hacen planes vocacionales más acordes con sus intereses y preferencias.

Nuestros resultados diferenciales, atendiendo a la variable género, coinciden con los de García-Montalvo (2001) quien encuentra superioridad de los hombres en algunos aspectos como en el menor tiempo para la obtención de empleo, superioridad de las mujeres en otros aspectos como la frecuencia e intensidad de la búsqueda de empleo y, semejanzas entre ambos géneros, en aspectos como formas de búsqueda de empleo, o procedimientos por los cuales se encuentra el primer empleo.

Respecto a las diferencias en función de la variable tipo de titulación (licenciatura vs. diplomatura o ciclo largo vs. corto) nuestros hallazgos también coinciden con los de GarcíaMontalvo (2001) quien obtiene superioridad del estudiantado de ciclo corto en aspectos como mayor búsqueda de empleo, menor tiempo de obtención empleo, o mayor valoración de la personalidad del diplomado por parte del primer empleador para la contratación.

De cara a futuras investigaciones, sugerimos que para ahondar en el conocimiento del desarrollo vocacional de los jóvenes universitarios, necesitamos contar con mayor cantidad de estudios que nos permitan examinar las características generales y diferenciales de la conducta vocacional en las etapas de "establecimiento" y "mantenimiento" propuestas por Super (1953), en las cuales los universitarios se encuentran ya plenamente integrados en el mercado laboral.

Coincidimos finalmente con Rivas (2007) en su crítica y escepticismo, respecto a la situación deficiente en la que se encuentra la investigación sobre la conducta y el asesoramiento vocacional, en un Estado, como el Español, que estando a la cola de la Unión Europea (UE), dedica tan solo el 4.5\% de su PIB (Producto Interior Bruto) a la educación, frente al 6\% europeo, o el 1.1\% a I+D (Investigación + Desarrollo), frente al 2.4\% de la UE (Cabrera, Bethencourt, Álvarez y González, 2006). 


\section{Referencias}

Álvarez, P., Bethencourt, J. T. y Cabrera, L. (2000a). La transición al mercado laboral de los psicopedagogos: Estudio de las dos primeras promociones en la Universidad de La Laguna. Revista de Psicología General y Aplicada, 53(3), 535-547.

Álvarez, P., Feliciano, L., Cabrera, L., Bethencourt, J. T. y González, M. (2000b). Criterios para la elección de optativas en la enseñanza universitaria: implicaciones para la función tutorial del profesorado. Congreso de Orientación Educativa en las Universidades. Granada. Andalucía.

Álvarez, V. B. (1994). Programas de desarrollo vocacional para la educación secundaria. En V. García Hoz, La orientación en la educación institucionalizada: la formación ética (pp. 366-391). Madrid: Rialp.

Bethencourt, J. T., Álvarez, P., Cabrera, L. y González, M. (2000). El proceso de laboralización de los psicopedagogos. Congreso de Orientación Educativa en las Universidades. Granada. Andalucía.

Bethencourt, J. T., González, M. C., Álvarez, P. y Cabrera, L. (2005). Conducta vocacional del estudiantado de la Universidad de La Laguna. Revista de Psicología General y Aplicada, 58(3), 379-395.

Cabrera, L., Bethencourt, J. T., González, M. C. y Álvarez, P. (2006). Un estudio transversal retrospectivo sobre prolongación y abandono de estudios universitarios. Relieve: Revista Electrónica de Investigación Educativa, 12(1), disponible online en http://www.uv.es/RELIEVE/v12n1/RELIEVEv12n1 1.htm

Cabrera, L., Bethencourt, J. T., Álvarez, P. y González, M. (2006). El problema del abandono de los estudios universitarios. Relieve: Revista Electrónica de Investigación Educativa, 12(2), disponible online http://www.uv.es/RELIEVE/v12n2/RELIEVEv12n2 1.htm

García-Montalvo, J (2001). Formación y empleo de los graduados de enseñanza superior en España y Europa. Valencia: Bancaja e IVIE (Instituto Valenciano de Investigaciones Económicas).

González, M. C., Álvarez, P., Cabrera, L. y Bethencourt, J. T. (2002). La toma de decisiones académicas del estudiantado de la Universidad de La Laguna en la elección de créditos de libre configuración. Contextos Educativos, 5, 123-139.

Rísquez, A. (2004). Efectos de las diferencias interculturales sobre el desarrollo de la carrera y la orientación vocacional: un estudio empírico de los factores que influyen en la mo- 
tivación hacia el trabajo de trabajadores irlandeses y españoles. Revista Española de Orientación y Psicopedagogía, 15(1), 11-31.

Rivas, F. (2007). ¿Conducta y asesoramiento vocacional en el mundo de hoy? Electronic Journal of Research in Educational Psychology, 5(1), 5-14.

Rocabert, E. (2003). Desarrollo vocacional. En F. Rivas, Asesoramiento vocacional. Teoría, práctica e instrumentación (pp. 201-236). Barcelona: Ariel.

Rocabert, E., Rivas, F. y Pascual, F. (2000). Análisis de los planes de estudio del grupo vocacional deportivo: una aproximación desde el desarrollo vocacional. Revista GalegoPortuguesa de Psicoloxía e Educación: Revista de Estudios e Investigación en Psicología y Educación, 5, 129-142.

Rodríguez, M. L. (1995). Educación para la carrera y diseño curricular. Teoría y práctica de programas de educación para el trabajo. Barcelona: Universitat de Barcelona

Salvador, A. y Peiró, J. M. (1986). La madurez vocacional. Evaluación, desarrollo y programas de intervención. Madrid: Alhambra.

Super, D. E. (1953). A theory of vocational development. American Psychologist, 8(5), 185190.

Super, D. E. (1980). A life-span, life-space approach to career development. Journal of Vocational Behavior, 16(3), 282-296.

Salomone, P. R. (1996). Tracing Super's theory of vocational development: a 40-year retrospective. Journal of Career Development, 22(3), 167-184.

Valls, F. y Martínez, J. M. (2004). Uso del inventario de desarrollo vocacional (CDI) de Super en España. Revista de Psicología General y Aplicada, 57(4), 463-476. 